\title{
Falla renal aguda asociada a síndrome de hiperestimulación ovárica
}

\author{
Acute renal failure associated with ovarian \\ hyperstimulation syndrome
}

\author{
John Fredy Nieto, Mónica Zuluaga, Arbey Aristizábal, \\ Lina María Serna, Catalina Ocampo, Gustavo Adolfo Zuluaga \\ - Medellín (Colombia)
}

\section{Resumen}

El síndrome de hiperestimulación ovárica es una complicación frecuente que se puede presentar en pacientes que reciben tratamientos de inducción de la ovulación y como consecuencia pueden desarrollar un síndrome edematoso grave con falla de uno o varios órganos; presentamos el caso de una paciente con falla renal aguda asociada a síndrome de hiperestimulación ovárica. (Acta Med Colomb 2016; 41:58-61).

Palabras clave: fertilización in vitro; síndrome de hiperestimulación ovárica; síndrome edematoso; falla renal aguda.
Abstract
Ovarian hyperstimulation syndrome is a common complication that can occur in patients receiving treatment for ovulation induction and as a consequence may develop a severe edematous syndrome with failure of one or more organs; the case of a patient with acute renal failure associated with ovarian hyperstimulation syndrome is presented. (Acta Med Colomb 2016; 41:58-61).
Keywords: in vitro fertilization, ovarian hyperstimulation syndrome, edematous syndrome, acute renal failure.

\author{
Dres: John Fredy Nieto-Ríos, Arbey Aristizábal \\ Alzate, Catalina Ocampo Kohn, Gustavo Adolfo \\ Zuluaga Valencia: Nefrólogos; Dra. Lina María \\ Serna Higuita: Nefróloga Pediatra. Hospital \\ Pablo Tobón Uribe, Universidad de Antioquia; \\ Dra. Mónica Zuluaga Quintero: Residente \\ Universidad Pontificia Bolivariana. Medellín \\ (Colombia). \\ Correspondencia. Dra. Lina María Serna Higui- \\ ta. Medellín (Colombia). \\ E-mail: johnfredynieto@gmail.com \\ Recibido: 27/III/2015 Aceptado: 29/I/2016
}

\section{Introducción}

Las terapias de inducción de la ovulación para la fertilización in vitro han aumentado notablemente en la última década. El síndrome de hiperestimulación ovárica es una entidad frecuente en pacientes que reciben tratamientos de inducción de la ovulación y como complicación asociada pueden desarrollar falla renal aguda. Presentamos el caso de una paciente que luego de la aplicación de medicamentos hormonales para inducir la ovulación desarrolla un síndrome edematoso grave y falla renal aguda asociado a síndrome de hiperestimulación ovárica.

\section{Reporte de caso}

Paciente de 36 años, sin ningún antecedente patológico de importancia, con diagnóstico de baja reserva ovárica y a quien le realizaron terapia de inducción de la ovulación con hormona gonadotropina coriónica humana (HGC) para fertilización in vitro. Mantenimiento hormonal con gesprogen ${ }^{\circledR}$ (progesterona intravenosa), utrogestan ${ }^{\circledR}$ (progesterona oral), progynova ${ }^{\circledR}$ (valerato de estradiol) y estrogel ${ }^{\circledR}$ (estradiol).
No antecedentes de consumo medicamentos diferentes a los prescritos; quince días después le realizan transferencia embrionaria. Posteriormente comenzó a presentar dolor lumbar, edema progresivo de miembros inferiores, abdomen y cara; además hiporexia, náuseas, vómito, oliguria, cefalea y visión borrosa. Al ingreso se documentó cifras de presión arterial elevadas y anasarca. Los exámenes mostraron elevación aguda de azoados (Tabla 1); la ecografía abdominal reportó ascitis, hepatomegalia y derrame pleural bilateral y riñones de tamaño normal con aumento de la ecogenicidad sin hidronefrosis; ecografía transvaginal útero en anteversión de 7.4 × 3.8 × $5.1 \mathrm{~cm}$, ovarios con múltiples folículos, algunos con signos de transformación hemorrágica, ovario derecho de $52 \times 32 \times 30 \mathrm{~mm}$ y ovario izquierdo de 55 × 34 x $32 \mathrm{~mm}$; dopppler de arterias renales normal; niveles de BHCG, estradiol y progesterona elevados; uroanálisis normal con sedimento inactivo, eosinófilos en orina negativos, proteinuria en orina de 24 horas de $140 \mathrm{mg}$. Ecocardiograma sin alteraciones. ANA, ENAs, ANCAS y anticuerpos anticardiolipinas negativos. Otros laboratorios se reportan 
Tabla 1. Resultados de laboratorio

\begin{tabular}{|c|c|c|c|c|c|c|c|c|}
\hline Citoquímico de orina & $\begin{array}{l}\text { Aspecto } \\
\text { Turbio }\end{array}$ & $\begin{array}{c}\text { pH } \\
6\end{array}$ & $\begin{array}{c}\text { Densidad } \\
1.010\end{array}$ & $\begin{array}{c}\text { Proteinuria } \\
0\end{array}$ & $\begin{array}{c}\text { Glucosuria } \\
0\end{array}$ & $\begin{array}{c}\text { Eosinofilos orina } \\
\text { Negativo }\end{array}$ & $\begin{array}{l}\text { Leucocitos } \\
0-5 \times \mathrm{mm}^{3}\end{array}$ & $\begin{array}{c}\text { Bacterias } \\
\quad \text { No }\end{array}$ \\
\hline Gases arteriales & $\begin{array}{c}\mathbf{p H} \\
7.37\end{array}$ & $\begin{array}{c}\mathbf{P a O}_{2} \\
69 \mathrm{mmHg}\end{array}$ & $\begin{array}{c}\mathrm{HCO}_{3} \\
18.5 \mathrm{mmol} / \mathrm{L}\end{array}$ & $\begin{array}{c}\mathbf{P C O}_{2} \\
32 \mathrm{mmHg}\end{array}$ & $\begin{array}{l}\mathrm{FiO}_{2} \\
0.21\end{array}$ & & & \\
\hline \multirow[t]{5}{*}{ Estudios séricos } & $\begin{array}{c}\text { Hb } \\
12.4 \mathrm{~g} / \mathrm{dl}\end{array}$ & $\begin{array}{c}\text { Hematocrito } \\
46 \%\end{array}$ & $\begin{array}{l}\text { Leucocitos } \\
11000 \mathrm{~mm}^{3}\end{array}$ & $\begin{array}{c}\text { Neutrofilos } \\
75.7 \%\end{array}$ & $\begin{array}{c}\text { Linfocitos } \\
15.2 \%\end{array}$ & $\begin{array}{c}\text { Eosinofilos } \\
1 \%\end{array}$ & $\begin{array}{c}\text { Monocitos } \\
5.9 \%\end{array}$ & $\begin{array}{c}\text { Plaquetas } \\
198.000 \mathrm{~mm}^{3}\end{array}$ \\
\hline & $\begin{array}{c}\text { VSG } \\
57 \mathrm{~mm} / \mathrm{h}\end{array}$ & $\begin{array}{l}\text { VCM } \\
88 \mathrm{fl}\end{array}$ & $\begin{array}{c}\text { TP } \\
11 \mathrm{seg}\end{array}$ & $\begin{array}{l}\text { INR } \\
1.01\end{array}$ & $\begin{array}{c}\text { TPT } \\
27.5 \mathrm{seg}\end{array}$ & \multicolumn{2}{|c|}{$\begin{array}{c}\text { Elecroforesis proteinas } \\
\text { Normal }\end{array}$} & \\
\hline & $\begin{array}{l}\text { Ácido Urico } \\
8.3 \mathrm{mg} / \mathrm{dL}\end{array}$ & $\begin{array}{c}\text { Lactato } \\
0.6 \mathrm{mmol} / \mathrm{L}\end{array}$ & $\begin{array}{l}\text { Creatinina } \\
3.35 \mathrm{mg} / \mathrm{dL}\end{array}$ & $\begin{array}{c}\text { BUN } \\
39.6 \mathrm{mg} / \mathrm{dL}\end{array}$ & $\begin{array}{l}\text { Albúmina } \\
3.2 \mathrm{~g} / \mathrm{dL}\end{array}$ & $\begin{array}{c}\text { Proteinas totales } \\
5.2 \mathrm{~g} / \mathrm{dL}\end{array}$ & $\begin{array}{l}\text { Glicemia } \\
97 \mathrm{mg} / \mathrm{dL}\end{array}$ & $\begin{array}{c}\text { PCR } \\
9.52 \mathrm{mg} / \mathrm{dL}\end{array}$ \\
\hline & $\begin{array}{c}\text { AST } \\
21 \mathrm{U} / \mathrm{L}\end{array}$ & $\begin{array}{c}\text { ALT } \\
16 \mathrm{U} / \mathrm{L}\end{array}$ & $\begin{array}{c}\text { FA } \\
75 \mathrm{U} / \mathrm{L}\end{array}$ & $\begin{array}{l}\text { GGT } \\
22 \mathrm{U} / \mathrm{L}\end{array}$ & $\begin{array}{c}\text { LDH } \\
193 \mathrm{U} / \mathrm{L}\end{array}$ & $\begin{array}{l}\text { Eosinufiflos } \\
\qquad 0.998\end{array}$ & $\begin{array}{l}\text { Monocitos } \\
1.49 \%\end{array}$ & $\begin{array}{l}\text { Plaguetas } \\
59.0000 \mathrm{~mm}^{2}\end{array}$ \\
\hline & $\begin{array}{c}\text { Sodio } \\
141 \mathrm{mmol} / \mathrm{L}\end{array}$ & $\begin{array}{c}\text { Potasio } \\
4.2 \mathrm{mmol} / \mathrm{L}\end{array}$ & $\begin{array}{c}\text { Cloro } \\
107 \mathrm{mg} / \mathrm{dL}\end{array}$ & $\begin{array}{c}\text { Fosforo } \\
5.8 \mathrm{mg} / \mathrm{dL}\end{array}$ & $\begin{array}{l}\text { Magnesio } \\
2.3 \mathrm{mg} / \mathrm{dL}\end{array}$ & $\begin{array}{c}\text { Calcio } \\
8.5 \mathrm{mg} / \mathrm{dL}\end{array}$ & $\begin{array}{c}\text { Calcio ionizado } \\
0.8 \mathrm{mmol} / \mathrm{L}\end{array}$ & $\begin{array}{c}\text { Ck total } \\
50 \mathrm{U} / 1\end{array}$ \\
\hline Estudios hormonales & $\begin{array}{c}\text { BHCG } \\
4297 \mathrm{mUI} / \mathrm{mL}\end{array}$ & $\begin{array}{c}\text { TSH } \\
6.39 \mathrm{mUI} / \mathrm{mL}\end{array}$ & $\begin{array}{c}\text { T3 } \\
1.18 \mathrm{ng} / \mathrm{dL}\end{array}$ & $\begin{array}{c}\text { T4L } \\
1.05 \mathrm{ng} / \mathrm{mL}\end{array}$ & $\begin{array}{c}\text { РТн } \\
71 \mathrm{pg} / \mathrm{mL}\end{array}$ & $\begin{array}{l}\text { Estradiol } \\
767 \mathrm{pg} / \mathrm{mL}\end{array}$ & $\begin{array}{c}\text { Progesterona } \\
49.12 \mathrm{ng} / \mathrm{mL}\end{array}$ & \\
\hline Estudios inmunológicos & $\begin{array}{c}\text { C3 } \\
132 \mathrm{mg} / \mathrm{dL}\end{array}$ & $\begin{array}{c}\text { C4 } \\
26,6 \mathrm{mg} / \mathrm{dL}\end{array}$ & $\begin{array}{c}\text { Anas } \\
\text { Negativo }\end{array}$ & $\begin{array}{l}\text { Anti DNA } \\
\text { Negativo }\end{array}$ & $\begin{array}{c}\text { ENAS } \\
\text { Negativos }\end{array}$ & $\begin{array}{c}\text { Anticardiolipinas } \\
\text { IgM e IgG negativo }\end{array}$ & $\begin{array}{c}\text { Ac AM } \\
\text { negativos }\end{array}$ & $\begin{array}{l}\text { ANCAS } \\
\text { negativos }\end{array}$ \\
\hline Estudios infecciosos & $\begin{array}{c}\text { VIH } \\
\text { Negativo }\end{array}$ & $\begin{array}{c}\text { Ac Hepatitis C } \\
\text { Negativo }\end{array}$ & $\begin{array}{l}\text { Ag S Hep B } \\
\text { Negativo }\end{array}$ & $\begin{array}{c}\text { VRDL } \\
\text { No reactivo }\end{array}$ & & & & \\
\hline Estudios urinarios & \multicolumn{2}{|c|}{$\begin{array}{l}\text { Proteínas orina de } \mathbf{2 4} \text { horas } \\
\qquad 140 \mathrm{mg} / \text { día }\end{array}$} & \multicolumn{2}{|c|}{$\begin{array}{l}\text { Depuracion creatinina medida } \\
35 \mathrm{~mL} / \mathrm{min} / 1.73 \mathrm{~m}^{2} \mathrm{Vol} 4050 \mathrm{~mL}\end{array}$} & $\begin{array}{l}\text { Sodio en orina } \\
<20 \mathrm{mmol} / \mathrm{L}\end{array}$ & & & \\
\hline $\begin{array}{l}\text { Hb: hemoglobina; VSG: } \\
\text { ALT: alanino aminotransfe } \\
\text { ENAS: anticuerpos contra }\end{array}$ & sedimentación & $\begin{array}{l}\text { merular; VCM: } \\
\text { GT: gamaglutan } \\
\text { iclo Anti RNP, S }\end{array}$ & insferasa; LD & tato deshidrog & asa; Ac AM: anti & $\begin{array}{l}\text { T: aspartato aminotran } \\
\text { cicuerpos antimicrosom } \\
\text { de los neutrófilos. }\end{array}$ & $\begin{array}{l}\text { isferasa, } \\
\text { ales; }\end{array}$ & \\
\hline
\end{tabular}

en la Tabla 1. Se diagnostica lesión renal aguda AKI 3 secundaria a síndrome de hiperestimulación ovárica (SHO). Se realizó tratamiento con restablecimiento del volumen circulatorio efectivo con cristaloides y albúmina, heparina profiláctica para prevención de eventos tromboembólicos, diuréticos y antihipertensivos (nifedipino, clonidina). El edema resolvió y dos semanas posterior al inicio del tratamiento la paciente recuperó completamente su función renal (Figura 1); las cifras de presión arterial se normalizaron y se lograron desmontar los antihipertensivos. Semanas después se documentó embarazo gemelar que culminó sin otras complicaciones.

\section{Discusión}

Presentamos el caso de una paciente previamente sana, que desarrolla un síndrome edematoso grave y falla renal aguda (FRA) luego de la administración de medicamentos hormonales para estimular la ovulación. Realizamos un estudio exhaustivo pero conservador en búsqueda de la causa de la falla renal. Luego de todos éstos concluimos que la paciente tenía una FRA asociada a SHO que evolucionó satisfactoriamente con el tratamiento instaurado.

El SHO es una complicación de las terapias de inducción de la ovulación para la fertilización in vitro, caracterizado por la presencia de edema progresivo y compromiso del

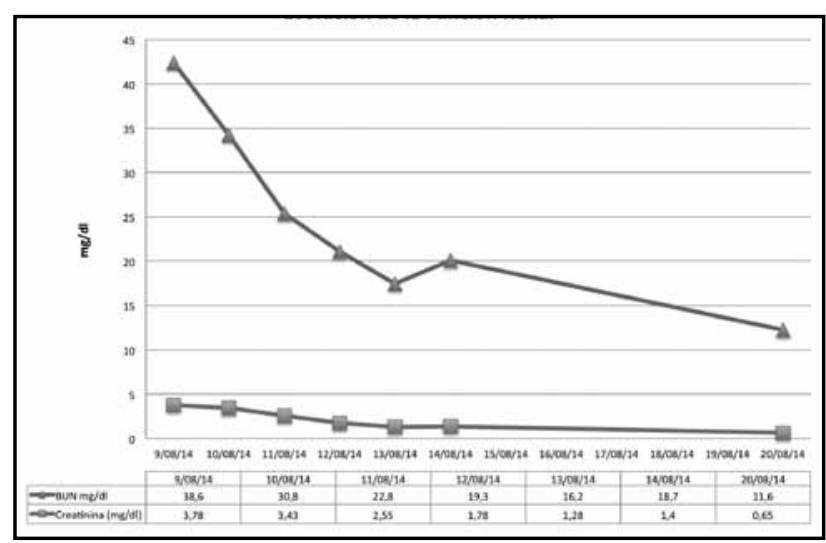

Figura 1. Evolución de la función renal. 
volumen circulatorio efectivo que puede llevar a múltiples complicaciones asociadas como FRA, falla cardiaca, crisis hipertensiva y trombosis en diversos lechos vasculares (1). Tiene una incidencia variable que va de 0.5 - $5 \%$ cuando se tienen en cuenta los casos graves, pero que puede llegar hasta el $33 \%$ en cuadros leves, con una mortalidad que alcanza 1 en 50000 pacientes $(1,2)$; como factores de riesgo se han identificado: la edad menor de 35 años, el bajo índice de masa corporal, niveles de estradiol mayores de $4000 \mathrm{pg} / \mathrm{mL}$, el desarrollo de más de ocho folículos en un ovario, antecedentes de síndrome de ovario poliquístico, mutaciones de la hormona folículo estimulante (FSH) y antecedente previo de SHO; sin embargo, también se han descrito casos espontáneos durante el embarazo, sobre todo asociado a gestaciones múltiples y enfermedad molar (1). No es clara hasta ahora la fisiopatología de este síndrome; algunos autores proponen que es secundario a la administración de la HCG para la estimulación ovárica, que incrementa la expresión del ácido ribonucleico (ARN) mensajero para el factor de crecimiento endotelial vascular (VEGF) y el receptor de VEGF 2, lo que conlleva a un aumento en la permeabilidad vascular, extravasación de proteínas al espacio intersticial y depleción del volumen intravascular; esto genera un desequilibrio hemodinámico y electrolítico con la consecuente aparición de tercer espacio (ascitis, derrame pleural y pericárdico) y edemas progresivos hasta un estado de anasarca y choque hipovolémico (3). Aunque el VEGF es el mayor protagonista, también se han implicado otras sustancias vasoactivas como interleuquinas: IL-1, IL-2, IL-6, IL-8, endotelina 1, factor de necrosis tumoral alfa y prostaglandinas que están asociadas con una vasodilatación arteriolar simultánea y la ocurrencia de disfunción circulatoria hiperdinámica que activa al sistema nervioso simpático, sistema renina angiotensina aldosterona y hormona antidiurética $(3,4)$.

Las manifestaciones clínicas en la mayoría de casos son leves, presentándose con síntomas generales, distensión abdominal, náuseas, vómito y edemas; sin embargo, puede evolucionar a un cuadro más grave con hipotensión o hipertensión, hipovolemia, desórdenes electrolíticos y un síndrome edematoso con franca anasarca. Se puede complicar con restricción en la dinámica ventilatoria y en algunos casos hidrotórax, atelectasias, falla respiratoria, FRA, falla cardiaca, falla hepática y alto riesgo de eventos tromboembólicos debido a la mayor hemoconcentración, aumento en la viscosidad plasmática, éstasis por inactividad física y disminución del flujo vascular; todo esto exige estar alerta ante síntomas sugestivos de trombosis venosa profunda o incluso en sitios inusuales $(1,2,5)$.

La gravedad del SHO está determinada por los parámetros clínicos y de laboratorio. El compromiso es leve cuando el tamaño de los ovarios es menor de $5 \mathrm{~cm}$, existe dolor abdominal de baja intensidad asociado a síntomas generales e incremento en niveles de estradiol y progesterona; moderado, si el tamaño ovárico está entre 5 y $10 \mathrm{~cm}$ y empeoramiento de los síntomas iniciales; y grave, cuando los ovarios alcanzan un diámetro mayor de $10 \mathrm{~cm}$ y/o existe falla de uno o varios órganos, que puede progresar en algunos casos a un estado crítico con desarrollo de choque hipovolémico y síndrome de dificultad respiratoria aguda (SDRA) $(1,2)$. El caso de nuestra paciente fue clasificado como grave dado el compromiso edematoso grave y la FRA.

La afección renal es frecuente, manifestándose con una lesión renal aguda de novo o el deterioro de un problema renal crónico de base $(5,6)$. La explicación a este fenómeno está dada por un compromiso de la perfusión renal secundario a la depleción de volumen vascular efectivo; pero contribuye a ello además, el grado de ascitis que puede producir un síndrome compartimental abdominal generando efecto obstructivo y resistencia al flujo (7). Esta última teoría es favorecida por algunos reportes descritos hasta ahora, donde la paracentesis terapéutica juega un papel importante en liberar la ascitis a tensión y por ende mejorar la falla renal $(8,9)$. Levin y colaboradores realizaron un estudio de intervención retrospectivo en 30 mujeres que desarrollan SHO grave y compararon los cambios en el gasto urinario y dinámica vascular antes y posterior a la realización de paracentesis evacuante; ellos demostraron un efecto aislado en la mejoría de la función renal que se evidencia por aumento en el gasto urinario, reducción de los azoados y descenso en el hematocrito (9) Maslovitz y colaboradores confirmaron lo mismo en un estudio similar realizado en 19 mujeres donde miden además la presión intraabdominal y la resistencia en el doppler de arterias renales; ellos reportan el aumento del gasto urinario, descenso en azoados y disminución en las resistencias vasculares y presión intraabdominal luego de la paracentesis (10). No obstante, la única explicación fisiopatológica de esta condición no es prerrenal, en la literatura se han descrito casos de uropatía obstructiva secundaria a la hiperproliferación ovárica lo que ocasiona presión sobre estructuras adyacentes entre ellas el uréter, con el desarrollo de hidronefrosis secundaria y lesión renal aguda posrenal (11-13). En nuestra paciente no fue necesaria la realización de paracentesis evacuante.

El diagnóstico diferencial de la FRA en estos casos incluye la nefritis tubulointersticial por medicamentos, la rabdomiolisis, la glomerulonefritis aguda, el síndrome nefrótico, el síndrome cardiorrenal, la trombosis de vasos renales y la enfermedad renal crónica no diagnosticada previamente. A nuestra paciente se le realizó un buen interrogatorio buscando causas medicamentosas o tóxicas y se efectuaron los estudios correspondientes con los cuales se descartaron estas entidades. No fue necesario realizarle biopsia renal dado el cuadro clínico claro que presentó la paciente con la buena evolución posterior y porque este procedimiento invasivo tiene riesgos altos en una mujer que estaba buscando embarazo.

El tratamiento dependerá de la gravedad del cuadro clínico y hallazgos de laboratorio $(1,2)$. Todos los casos graves deben tener manejo intrahospitalario incluyendo cuadros que se presenten con dolor abdominal intenso, 
vómito incontrolable, deshidratación, ascitis a tensión y disnea. El manejo básico consiste en el control de los síntomas, analgesia idealmente con acetaminofén y opioides; evitar el uso de antiinflamatorios no esteroideos, hidratación con cristaloides y de ser necesario reposición de albúmina, soporte inotrópico, monitoreo de la presión venosa central y gasto urinario, corrección de desórdenes electrolíticos, tromboprofilaxis con heparinas de bajo peso molecular o heparina no fraccionada, nunca warfarina por la probabilidad de gestación, control de complicaciones y si es necesario en casos refractarios o con síntomas graves, paracentesis evacuante y terapia de reemplazo renal; sin embargo, en la mayoría de casos hay autorresolución del cuadro paralelo con el descenso de los niveles de HCG y mejoría del compromiso sistémico $(1,5,6)$. Generalmente los síntomas leves desaparecen en una semana y cuadros más graves pueden demorar hasta dos a tres semanas en resolver (2).

Finalmente el objetivo y propuesta para esta condición es identificar siempre aquellas mujeres con factores de riesgo para desarrollar SHO y estar alerta a la evolución y complicaciones, con especial atención en aquellas condiciones que pueden acentuar los efectos sistémicos y tomar medidas de prevención y manejo oportunas en ciertos casos, evitando su progresión a un estado crítico (5). Puesto que el procedimiento de fertilización in vitro es ambulatorio, se debe realizar un seguimiento clínico y de ser necesario paraclínico de manera estricta para detectar esta complicación precozmente. Igualmente los clínicos distintos al área de la ginecología deben conocer que existe esta complicación con el fin de apoyar al ginecólogo en su manejo.

Aún quedan muchas preguntas por resolver acerca de esta condición, su fisiopatología no está completamente dilucidada y las estrategias para predecir y prevenir su desarrollo continúan en estudio.

\section{Conclusión}

La FRA asociada a síndrome de hiperestimulación ovárica es un diagnóstico a considerar en pacientes sometidas a tratamientos hormonales para estimular la ovulación.
En los casos graves el manejo debe ser multidisciplinario y conservador para proteger la vida de la mamá y de sus embriones. El conocimiento de este síndrome ayuda a los clínicos a evitar errores en la práctica clínica, pensando en otros diagnósticos que pueden llevar a terapias inadecuadas.

\section{Declaración de interés}

Los autores declaran no tener ningún conflicto de interés sobre el contenido de este artículo.

\section{Referencias}

1. Barry Hahn. ovarian hyperstimulation syndrome. J Emerg Med. 2007; 33: 191-192.

2. Rutkowski A, Dubinsky I. Ovarian hyperstimulation syndrome: imperatives for the emergency physician. J Emerg Med. 1999; 17: 669-672.

3. Balasch J, Arroyo V, Fabregues F, Salo J, Jimenez W, Pare JC et al. Neurohormonal and Hemodynamic Changes in Severe Cases of the Ovarian Hyperstimulation Syndrome . Ann Intern Med. 1994; 121: 27-33.

4. Shawaf TA, Grudzinskas G. Prevention and treatment of ovarian hyperstimulation syndrome. Best Pract Resear Clin Obst Gynaeco. 2003; 17: 249-261.

5. Shmorgun D, Claman O, Ottawa. The diagnosis and management of ovarian hyperstimulation síndrome. J Obstet Gynaecol Can. 2011; 33: 1156-1162.

6. Budev MM, Arroliga AC, Falcone T. Ovarian hyperstimulation syndrome. Crit Care Med. 2005; 33 (10 Suppl): S301-S306.

7. Grossman L, Konstantinos BA, Michalakis G, Browne H, Payson M, Segars J. The pathophysiology of ovarian hyperstimulation syndrome: an unrecognized compartment síndrome. Fertil Steril. 2010; 94.

8. Chen CD, Yang JH, Chao KH, Chen SU, Ho HN, Yang YS. Effects of repeated abdominal paracentesis on uterine and intraovarian haemodynamics and pregnancy outcome in severe ovarian hyperstimulation syndrome. Hum Reprod. 1998; 13: 2077-81

9. Levin I, Pharm B, Almog B, Avni A. Effect of paracentesis of ascitic fluids on urinary output and blood indices in patients with severe ovarian hyperstimulation syndrome. Fertil Steril. 2002; 77: 986-8.

10. Maslovitz S, Jaffa A, Eytan O, Wolman I, Many A, Lessing J. Renal Blood Flow Alteration After Paracentesis in Women With Ovarian Hyperstimulation. Obstet Gynecol. 2004; 104: 321-6.

11. Marak CP, Chopra A, Alappan N, Ponea A, Guddati AK. Ovarian Hyperstimulation Syndrome as an Etiology of Obstructive Uropathy. Case Repo ObsteGynecol. 2013, Article ID 653704, 3 pages.

12. Wiygul J, lallas $\mathbf{C}$, silverstein A, polascik T. Ovarian hyperstimulation causing ureteral obstruction in a pregnant woman. Urology. 2006; 67: 1085.e5-1085.e6.

13. Merrilees D, Smith A, Robinson R. Obstructive uropathy caused by OHSS Fertil Steril. 2008; 89: 992. 